\title{
DETERMINATION OF FAULT PLANE SOLUTIONS USING WAVEFORM AMPLITUDES AND RADIATION PATTERN
}

\author{
Vamvakaris, D.A., Papazachos, C.B., Karagianni, E.E., Scordilis, E.M. and \\ Chatzidimitriou P.M. \\ Geophysical Laboratory, School of Geology, Aristotle University of Thessaloniki, PO Box 352-1 \\ 54124, Thessaloniki-GREECE, dom@lemnos.geo.auth.gr
}

\section{ABSTRACT}

In the present work a modified version of the program FPFIT (Reasenberg and Oppenheimer, 1985 ) is developed, in order to improve the calculation of the fault plane solutions. The method is applied on selected earthquakes from short period waveform data in the Mygdonia basin ( $\mathrm{N}$. Greece) as recorded by the permanent network of the Seismological Station of Aristotle University of Thessaloniki during the period 1989-1999.

The proposed modification of the FPFIT program was developed in order to minimize the derivation of multiple solutions, as well as the uncertainties in the location of $\mathrm{P}$ and $\mathrm{T}$ axis of the determined fault plane solutions. Compared to the original version of FPFIT the modified approach takes also into account the radiation pattern of SV and $\mathrm{SH}$ waves. For each earthquake horizontal and vertical components of each station were used and the first arrivals of $\mathrm{P}$ and $\mathrm{S}$ waves were picked. Using the maximum peak-to-peak amplitude of $P$ and $S$ waves the ratio $P \max /\left(S N^{2} \max +S E^{2} \max \right)^{1 / 2}$ was estimated, where SNmax and SEmax are the maximum amplitudes of the two horizontal components (N-S, E-W) for the $S$ waves and Pmax is the maximum amplitude of the vertical one for the P- waves. This ratio for the observed data, as well as the corresponding ratio $P \mathrm{rad} /\left(S H^{2} \mathrm{rad}+S V^{2} \mathrm{rad}\right)^{1 / 2}$ of the synthetic data was used as a weight for the determination of the observed and theoretical P-wave polarities, respectively.

The method was tested using synthetic data. A significant improvement of the results was found, compared to the original version of FPFIT. In particular, an improved approximation of the input focal mechanism is found, without multiple solutions and the best-estimated $P$ and $T$ axes exhibit much smaller uncertainties. The addition of noise in the synthetic data didn't significantly change the results concerning the fault plane solutions. Finally, we have applied the modified program on a real data set of earthquakes that occurred in the Mygdonia basin.

\section{INTRODUCTION}

Focal mechanisms can provide useful information about structure and kinematics of faults and can else constrain the crustal stress field in which the earthquakes occur. The use of P-wave firstmotion polarities is the most common method for the calculation of the fault plane solutions from short-period data. The spatial distribution of P-wave first-arrivals and their polarities (compression or dilatation) are used to determine the two nodal planes. Given an excellent station coverage for an earthquake in a region for which the earth structure is well determined and with well recorded impulsive P-wave first arrivals, a reliable estimate for the fault-plane solution can be obtained, However, the station density is often poor, the velocity structure is known only approximately and the polarities of the first arrivals are often ambiguous. Generally, the poorer the data, the larger the number of possible solutions.

S/P amplitude ratios have been also used (e.g. Kisslinger, 1980; Hardebeck and Shearer, 2003) to determine focal mechanisms. P-wave amplitudes are large near the maximum stress axes ( $P$ and $T$ ) of the focal mechanism and smaller near the nodal planes, so a systematic variation of P/S amplitude ratios is expected. The use of P-wave amplitudes at a station should be performed with corrections for several factors like geometrical spreading, attenuation, station site effects and event 
magnitude. These factors are essentially cancelled and their influence is minimized by the use of the $\mathrm{P} / \mathrm{S}$ amplitude ratios.

In order to test the efficiency of the use of amplitude ratios for the evaluation of fault plane solutions, we have investigated earthquakes that occurred in the Mygdonia basin. These events are recorded with relatively good station coverage, without noise and clipped amplitudes in the waveforms.

\section{METHOD}

In order to improve the results, the FPFIT program was used as a reference basis and modified in several critical points. Thus, the radiation pattern of both transverse SV and SH waves were also incorporated in the process of determination against the original version that takes into consideration only the radiation pattern of $\mathrm{P}$-waves.

The radiation pattern for $\mathrm{P}$-waves can be described as a product of six suitable factors $P C_{i}$ $(i=1,2, \ldots, 6)$ with the moment tensor components:

$$
M=\left(\begin{array}{lll}
M_{11} & M_{12} & M_{13} \\
M_{21} & M_{22} & M_{23} \\
M_{31} & M_{32} & M_{33}
\end{array}\right)
$$

This tensor is described by nine components in space, which correspond to six independent components, because of symmetry $\left(M_{12}=M_{21}, M_{13}=M_{31}\right.$ and $\left.M_{23}=M_{32}\right)$. Thus, the radiation on the seismic focus is calculated for $\mathrm{P}$-waves from the sum

$$
\operatorname{Pr} a d=\sum_{i=1}^{6}\left(\mathrm{M}_{\mathrm{i}} * P c_{i}\right)
$$

where:

$$
\mathrm{M}_{1}=\mathrm{M}_{11}, \quad \mathrm{M}_{2}=\mathrm{M}_{12}, \quad \mathrm{M}_{3}=\mathrm{M}_{13}, \quad \mathrm{M}_{4}=\mathrm{M}_{22}, \quad \mathrm{M}_{5}=\mathrm{M}_{23}, \quad \mathrm{M}_{6}=\mathrm{M}_{33}
$$

The $P C_{i}$ values are a function of the take-off angle, $i$ and its azimuth, $\varphi$. Using the expressions of Aki and Richards (1980) that describes the moment tensor components as a function of fault strike, dip and rake, equation (2) becomes:

$$
\begin{aligned}
& \operatorname{Pr} a d=\cos \lambda \cdot \sin \delta \cdot \sin ^{2} i \cdot \sin 2(\varphi-\xi)-\cos \lambda \cdot \cos \delta \cdot \sin 2 i \cdot \cos (\varphi-\xi)+ \\
& +\sin \lambda \cdot \sin 2 \delta \cdot\left(\cos ^{2} i-\sin ^{2} i \cdot \sin ^{2}(\varphi-\xi)\right)+\sin \lambda \cdot \cos 2 \delta \cdot \sin 2 i \cdot \sin (\varphi-\xi)
\end{aligned}
$$

where: $\xi=$ strike

$$
\begin{aligned}
& \delta=\text { dip } \\
& \lambda=\text { rake } \\
& i=\text { take-off angle } \\
& \varphi=\text { take-off azimuth }
\end{aligned}
$$

Using a similar approach, the corresponding factors can be obtained for SV and SH waves, that can be multiplied by the moment tensor, $M_{1}$ elements using the well-known relations (Aki and Richards, 1980).

The radiation of seismic energy for $\mathrm{SV}$ waves is given by the equation:

$$
\begin{aligned}
& \text { SVrad }=\sin \lambda \cdot \cos 2 \delta \cdot \cos i \cdot \sin (\varphi-\xi)-\cos \lambda \cdot \cos \delta \cdot \cos 2 i \cdot \cos (\varphi-\xi)+ \\
& +\frac{1}{2} \cos \lambda \cdot \sin \delta \cdot \sin 2 i \cdot \sin 2(\varphi-\xi)-\frac{1}{2} \sin \lambda \cdot \cos 2 \delta \cdot \sin 2 i \cdot \sin (\phi-\xi)
\end{aligned}
$$

and respectively for the $\mathrm{SH}$ waves: 


$$
\begin{aligned}
& \text { SHrad }=\cos \lambda \cdot \cos \delta \cdot \cos i \cdot \sin (\varphi-\xi)+\cos \lambda \cdot \sin \delta \cdot \sin i \cdot \cos 2(\phi-\xi)+ \\
& +\sin \lambda \cdot \cos 2 \lambda \cdot \cos i \cdot \cos (\varphi-\xi)-\frac{1}{2} \sin \lambda \cdot \sin 2 \delta \cdot \sin i \cdot \sin 2(\varphi-\xi)
\end{aligned}
$$

In order to calculate the factors $S \mathrm{SC}_{i}$ and $S H c_{i}$ that should satisfy the relations for $\mathrm{SV}$ and $\mathrm{SH}$ waves, similar to the factors $P C_{i}$, equations (4) and (5) are written as:

$$
\begin{gathered}
\text { SVrad }=\sum_{i=1}^{6}\left(M_{i} * S V c_{i}\right) \\
\text { SHrad }=\sum_{i=1}^{6}\left(M_{i} * S H c_{i}\right)
\end{gathered}
$$

Here, the six values that form the variables $P_{C}, S V_{C}$ and $S H c$ are trigonometrical factors of the take-off angle, $i$, or even the azimuth, $\varphi$ and respectively are:

$$
\begin{array}{lll}
P c_{1}=\cos ^{2} i & S V c_{1}=0 & S H c_{1}=-\frac{1}{2} \sin 2 i \\
P c_{2}=\sin 2 i \cdot \cos \varphi & S V c_{2}=-\sin \varphi \cdot \cos i & S H c_{2}=\cos \varphi \cdot \cos 2 i \\
P c_{3}=\sin ^{2} i \cdot \cos ^{2} \varphi & S V c_{3}=-\frac{1}{2} \sin 2 \varphi \cdot \sin i & S H c_{3}=\frac{1}{2} \sin 2 i \cdot \cos ^{2} \varphi \\
P c_{4}=-\sin 2 i \cdot \sin \varphi & S V c_{4}=-\cos \varphi \cdot \cos i & S H c_{4}=-\cos 2 i \cdot \sin \varphi \\
P c_{5}=-\sin ^{2} i \cdot \sin 2 \varphi & S V c_{5}=-\sin i \cdot \cos 2 \varphi & S H c_{5}=-\frac{1}{2} \sin 2 i \cdot \sin 2 \varphi \\
P c_{6}=\sin ^{2} i \cdot \sin ^{2} \varphi & S V c_{6}=\frac{1}{2} \sin 2 \varphi \cdot \sin i & S H c_{6}=\frac{1}{2} \sin 2 i \cdot \sin ^{2} \varphi
\end{array}
$$

Equations (8) were verified using synthetic examples for test fault planes, shown in Table (1) below.

Table 1: Parameters for several synthetic fault planes used for method testing

\begin{tabular}{c|llll}
\hline No & Strike $\left(\xi^{\circ}\right)$ & Dip $\left(\delta^{\circ}\right)$ & Rake $\left(\lambda^{\circ}\right)$ & Rupture type \\
\hline 1 & 90 & 45 & 90 & Thrust \\
2 & 90 & 45 & -45 & Oblique-Normal \\
\hline
\end{tabular}

The azimuthal distributions of the radiation for both $\mathrm{P}$ and $\mathrm{SV}$ waves for two cases, a thrust fault (No.1) and an oblique fault (No.2) are shown in figures (1) and (2). In both figures, the radiation pattern using the P-waves illustrates the location of the two planes where the value of the seismic radiation is close to zero (white color). High values of radiation describe the location of the maximum dilatation T-axis, while low values the location of the maximum compression $\mathrm{P}$-axis. Both high and low values (maximum absolute values) are illustrated with black color.

In figure 1 we can see that the maximum-minimum $\mathrm{P}$-radiation areas around the $\mathrm{P}$ and $\mathrm{T}$ axes show a rather large spread. For this reason, the ratios $P \mathrm{rad} / S V \mathrm{rad}$ and $\mathrm{Prad} /\left(S H^{2} \mathrm{rad}+S V^{2} \mathrm{rad}\right)^{1 / 2}$ were used using the equations described before. The second ratio was examined because through its use we can avoid the rotation of the 3D axis-system in the radial-transverse horizontal axis system, witch is necessary for the first ratio (P/SV) traditionally used (e.g. Kisslinger et al., 1981).

In order to compare the results for the two cases, the results produced by the use of Prad and the ratios $P r a d / S V r a d$ and $P r a d /\left(S H^{2} r a d+S V^{2} \text { rad }\right)^{1 / 2}$ are plotted in figure (2). In the top part of this 
figure (fig.2, a1 and a2) the radiation of $\mathrm{P}$-waves is plotted. The ratio of radiation of $\mathrm{P}$-waves, to that of SV-waves (Prad/SVrad) is presented in the central part of figure 2 (b1 and b2), while the ratio $\operatorname{Prad} /\left(S H^{2} \mathrm{rad}+S V^{2} \mathrm{rad}\right)^{1 / 2}$ in the bottom (c1 and $\left.\mathrm{c} 2\right)$. Comparing these three plots for the same fault plane we observe that the location of the $P$ and $T$ axes is much better constrained by the use of the ratio $\mathrm{Prad} /\left(\mathrm{SH}^{2} \mathrm{rad}+\mathrm{SV}^{2} \mathrm{rad}\right)^{1 / 2}$, against the exclusive use of radiation of $\mathrm{P}$-waves, where the dispersion of the possible stress axes location is relatively large. On the other hand, the ratio Prad/SV $\mathrm{rad}$ does not appear to provide more useful information about the location of the axes, in these cases.

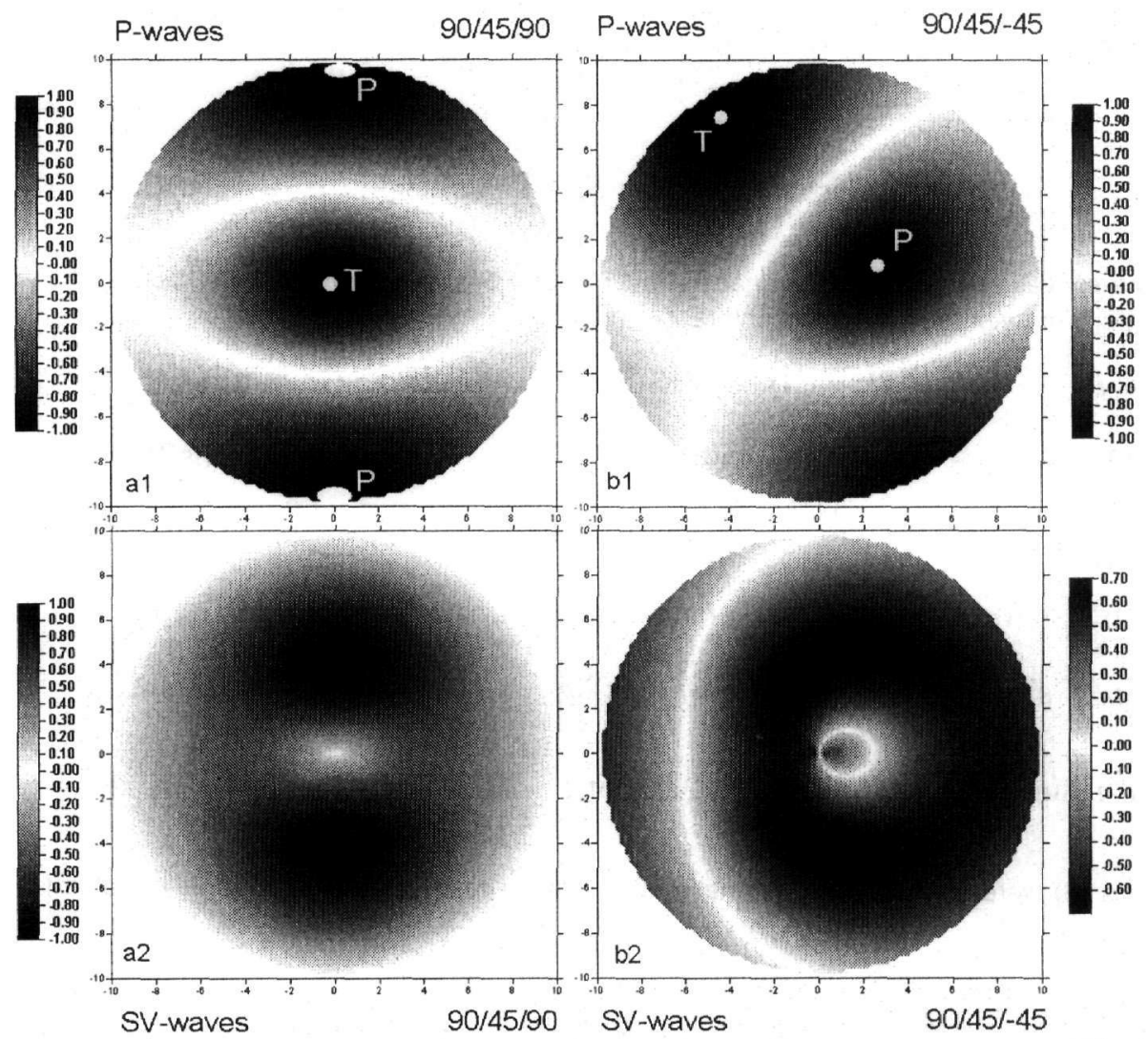

Figure 1: Distribution of the seismic radiation for the planes with parameters a) strike $=90^{\circ}, \operatorname{dip}=45^{\circ}$ and rake $=90^{\circ}$, and b) strike $=90^{\circ}$, dip $=45^{\circ}$ and rake $=-45^{\circ}$ by the use of 1) P-waves and 2) SV-waves.

The most common codes for the calculation of focal mechanisms, such as FPFIT (Reasenberg and Oppenheimer, 1985), find the source model that best fits a given set of observed first motion polarities. FPFIT uses all the information from waveforms of each station and presents graphically the distribution of first arrivals of P-waves for each earthquake. Taking into consideration this distribution of the first motions of P-waves, the program adopts suitably the two fault planes. The determination of the location of these two planes is realized by the separation of the compressional first motions from the dilatations. For this determination a multiple grid-search is used to find the double couple fault-plane solution minimizing a sum of first-motion polarity discrepancies:

$$
F=\sum_{k}\left\{\left|P_{k}^{o}-P_{k}^{o}\right| w^{k}\right\}
$$

In the minimization two weighted factors are incorporated in the weight factor, $w^{k}$. One of them reflects the estimated data variance (e.g. quality of the first-motion estimation) and the other is based on the theoretical radiation amplitude of P-waves (Aki and Richards, 1980), in order to reflect 
the lower quality of first motions near the nodal planes. Values for the observed P-radiation amplitudes, $\mathrm{P}^{\circ}$, depend just on the first-motion polarities and are 0.5 for compression and -0.5 for dilatation. A similar approach is also used for the theoretical radiation amplitude of $P$-waves, $P^{\text {th }}$.

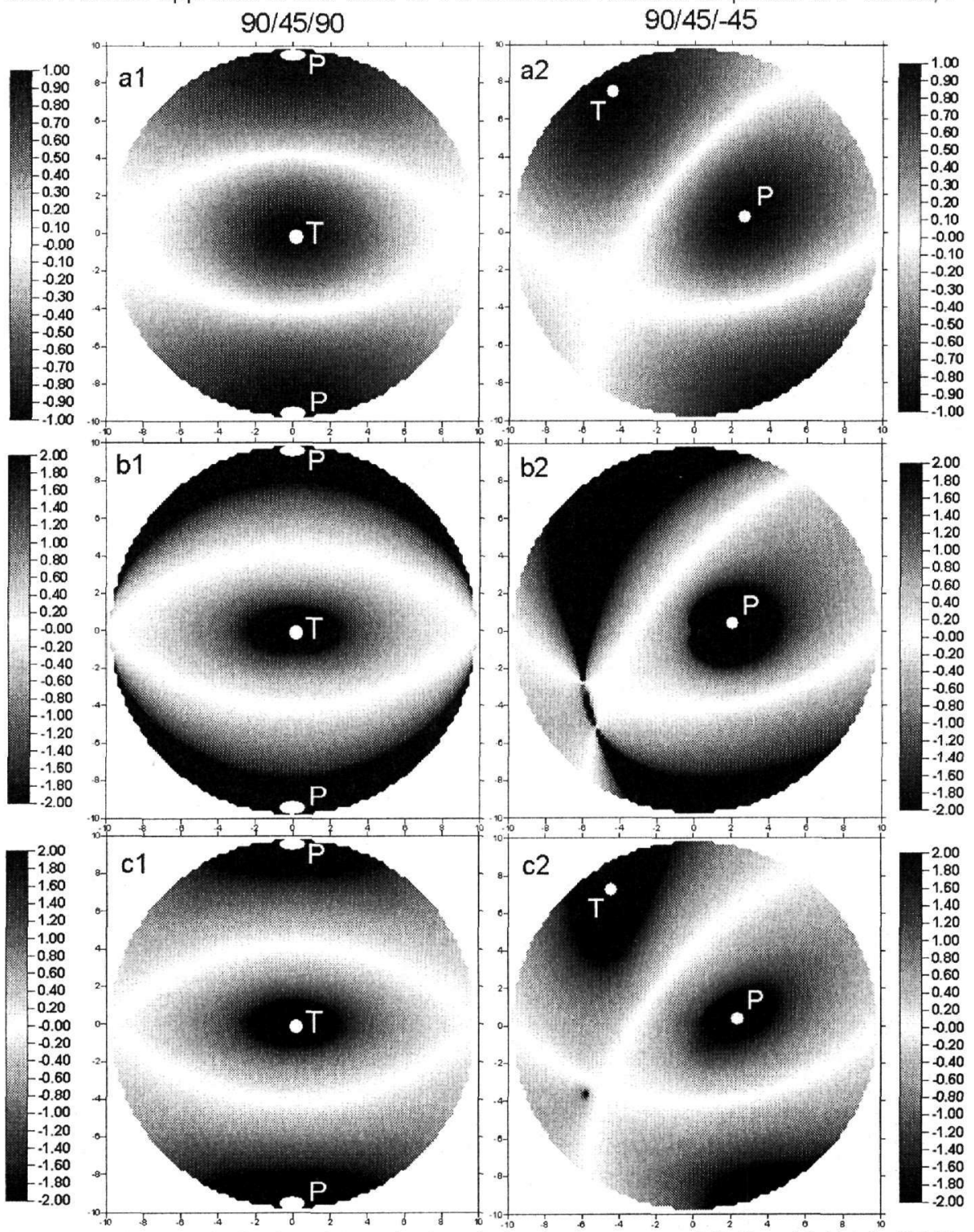

Figure 2: Azimuthal distribution of the seismic radiation for the ruptures 1 (left) and 2 (right) of Table (1). The results shown are produced by the use of a) the radiation pattern of P-waves (Prad), b) the ratio Prad/SVrad and, c) the ratio Prad/(SH $\left.{ }^{2} \mathrm{rad}+S V^{2} \mathrm{rad}\right)^{1 / 2}$

For the evaluation of the fault plane solution we have modified FPFIT in order to use ratios of $\mathrm{P} / \mathrm{S}$ waves. The ratio $P \max /\left(S N^{2} \max +S E^{2} \max \right)^{1 / 2}$ of the maximum amplitudes from the recorded waveforms was incorporated in the calculation of the modified misfit:

$$
F^{\mathrm{mod}}=\sum_{k}\left\{\left|\frac{P \max ^{k}}{\sqrt{S N \max _{k}^{2}+S E \max _{k}^{2}}}-\frac{\operatorname{Pr} a d^{k}}{\sqrt{S H r a d_{k}^{2}+S \operatorname{Vrad}_{k}^{2}}}\right| w^{k}\right\}
$$


In the proposed approach, the theoretical part is described by the ratio $\operatorname{Prad} /\left(S H^{2} \mathrm{rad}+S V^{2} \mathrm{rad}\right)^{1 / 2}$ of the radiation pattern for $\mathrm{P}, \mathrm{SH}$ and $\mathrm{SV}$ waves. Waveform amplitudes are used in order to calculate the ratio $P \max /\left(S N^{2} \max +S E^{2} \max \right)^{1 / 2}$, which describes the observed part of equation (10). The sum of the differences between these values for observed and theoretical values gives the misfit, $\mathrm{F}^{\text {mod }}$, which is used in the modified program. An advantage of the use of the modified misfit, $\mathrm{F}^{\text {mod }}$, is that amplitudes have a wide range of values, not the simple binary up or down (+ or -) value of the P-wave first motions (used in equation 9) and therefore the location of a given observation on the focal sphere can be constrained with a better precision. Furthermore, the contribution of $P$ and $S$ amplitudes permits also to include in the calculation of the focal mechanism the "nodal" or "zeroamplitude" arrivals.

\section{APPLICATION WITH REAL DATA}

Data for two earthquakes which occurred in the Mygdonia basin were selected (see Table 2) in order to evaluate the proposed method.

Table 2: Parameters for the two earthquakes used to test the proposed method.

\begin{tabular}{l|lllllllll}
\hline No & Date & Time & Lat & Lon & Dep & Mag & Strike & dip & Rake \\
\hline 1 & 19920409 & 054247.07 & 40.660 & 23.120 & 1.2 & 3.5 & 225 & 57 & -57 \\
2 & 19920502 & 094104.99 & 40.660 & 23.520 & 2.5 & 4.1 & 241 & 52 & -101 \\
\hline
\end{tabular}

The original FPFIT program was applied for these earthquakes and the determined fault planes (Table 2) were considered as real. In the next step, the focal mechanism were recalculated using the proposed modified version of FPFIT program, in order to test whether the modified approach is able to give the correct fault plane solution (which is a priori considered as correct). The results are shown in Figure 3 for the two earthquakes, where the fault planes as well as the position of the $\mathrm{P}$ and $\mathrm{T}$ axis are shown.

Comparison of the results for each earthquake using both versions of the program verifies that the proposed method revision provides the correct fault plane solution, as that was considered a priori. Moreover, significant decrease in the dispersion of the main stress axis ( $P$ and $T)$ is also observed for the modified approach FPFIT. However, for further testing of the proposed approach, we have chosen to remove a number of stations from the data set (one to four stations) selected either randomly or systematically (near or far from the nodal planes), as well as to insert an arbitrary noise level in the observed P/S amplitude ratios, in order to compare the stability of the obtained results with that of the complete and noiseless data. Several data removal tests were performed, namely:

I. Removal of one station "near" the plane for each earthquake

II. Removal of one station "far" from the plane for each earthquake

III. Removal of two stations "near" the plane for each earthquake

IV. Removal of two stations "far" from the plane for each earthquake

$V$. Removal of three random stations for each earthquake

VI. Removal of four random stations for each earthquake

Fault plane solutions were calculated for all these cases. Moreover, a pre-selected noise level, a, was inserted in the data. Noise level of $10 \%$ and $50 \%$ was tested for the examined six cases, by introducing randomly distributed errors in the observed $\mathrm{P} / \mathrm{S}$ ratios.

Figures 4 and 5 present some of the obtained results from all the cases that have been tested. In the first case (Fig. 4) earthquake No.2 of Table 2 was used, after removing data for the stations FNA and THE. These stations were selected to be removed because they were located "near" the fault planes. The original version (Fig. 4 a) provides a different solution from the one considered as real (Fig. 3c), contrary to the modified version (Fig. 4b), where almost no change is observed. The distribution of the $\mathrm{P}$ and $\mathrm{T}$ axis is also very well constrained by the proposed method. In the same figure the solutions of the proposed modified method with 10\% (Fig. 4C) and 50\% (Fig. 4d) noise are presented. It's clear that noise does not affect the results significantly, even if the case of $50 \%$ noise level of the P/S ratios. 


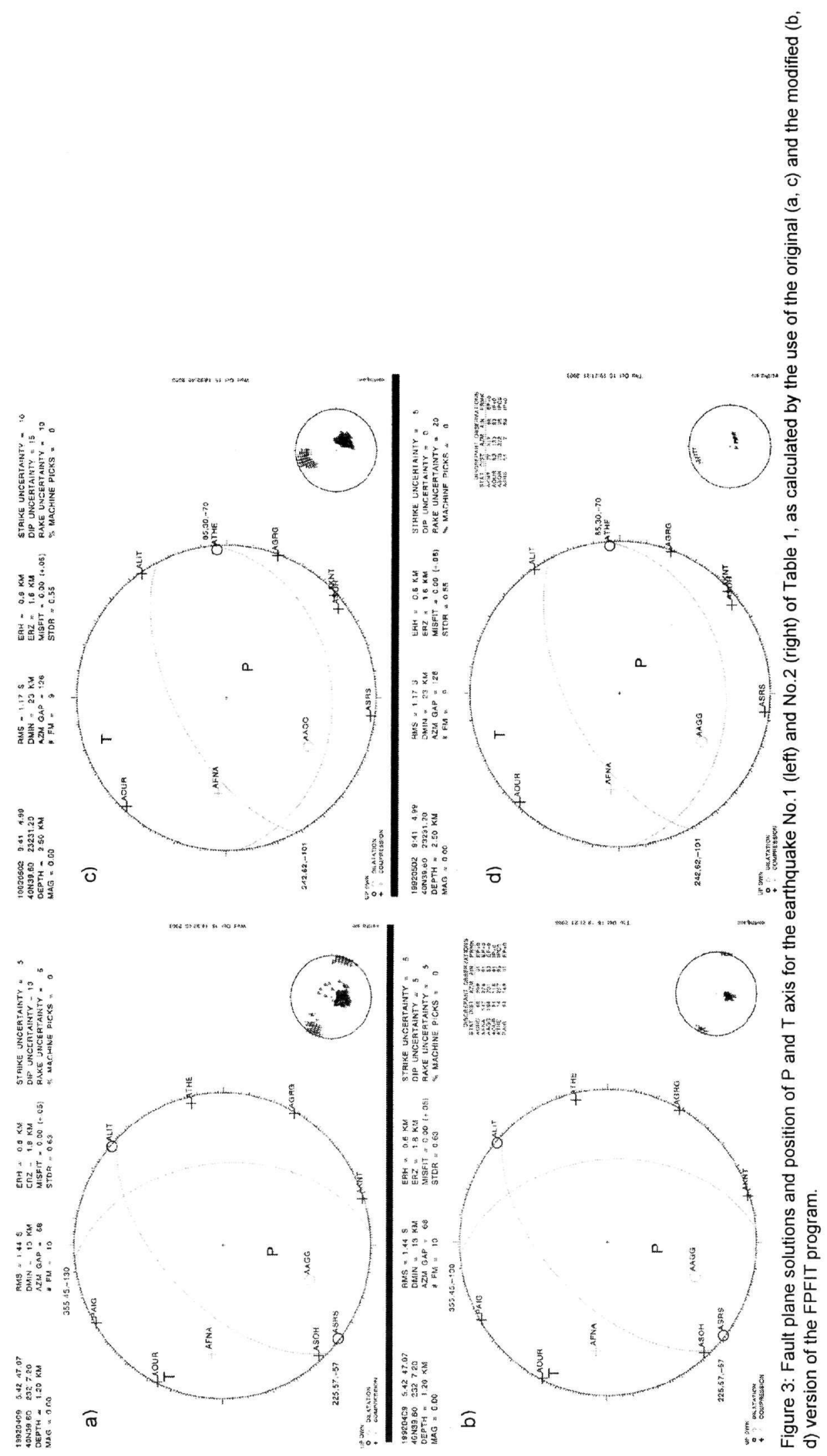




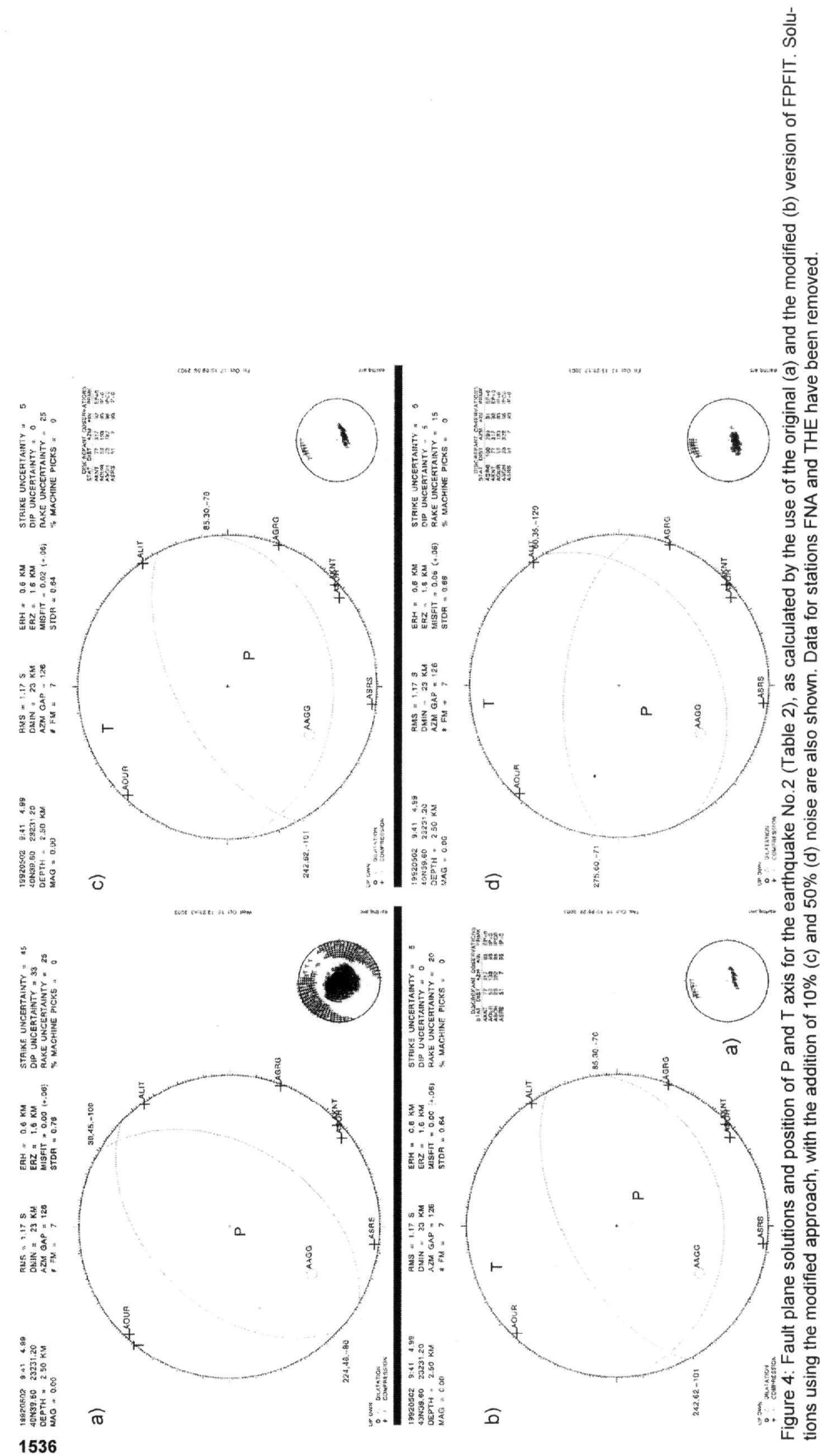




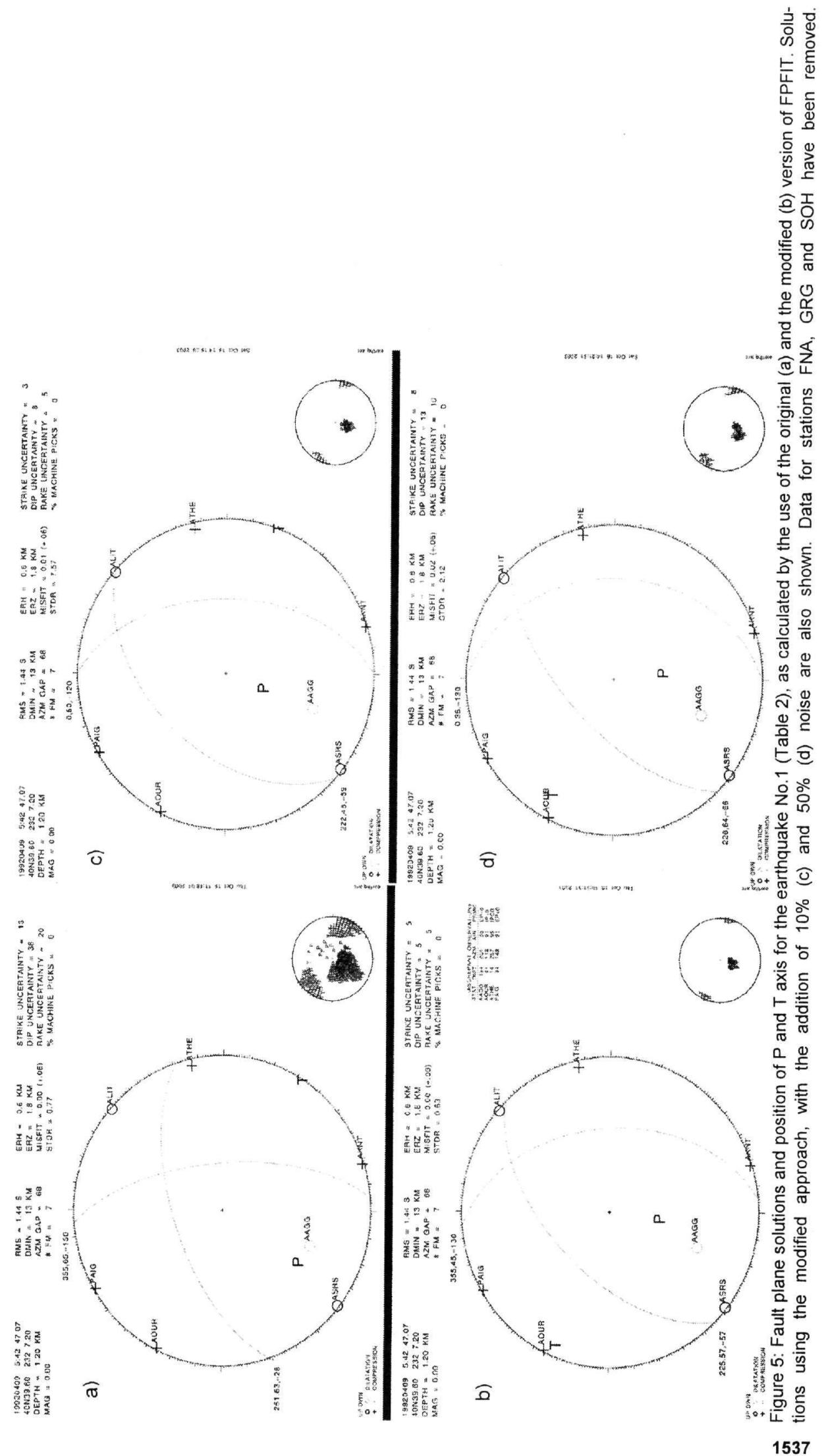


In figure 5, data for three stations (FNA, GRG and $\mathrm{SOH}$ ) were removed for earthquake No.1 of Table 2. In Figure 5a, we can observe a very different solution from the one considered (Fig. 3a), using the original FPFIT version. On the other hand, the proposed modified version provides a result that seems not to be affected by the lack of data but also by the noise (Fig. $5 \mathrm{c}$ and $5 \mathrm{~d}$ ).

\section{CONCLUSIONS}

We have confirmed that the use of waveform amplitudes and radiation pattern for both $\mathrm{P}$ and $\mathrm{S}$ waves gives P/S amplitude ratios that are generally consistent with the amplitude ratios expected from mechanisms based on P-wave polarity data. A modified version of FPFIT was developed in order to incorporate these P/S amplitude ratios. The improved focal mechanisms for events that occurred in the Mygdonia basin demonstrate the advantage of using amplitude and radiation ratios in focal mechanism inversion. The proposed approach does not require the rotation of the waveforms in the radial-transverse system, as the peak $\mathrm{S}$-wave amplitude is used in the process.

For the distribution of the main stress axes ( $P$ and $T)$, a large dispersion of their possible position is presented in the original first-motion version, contrary to the proposed modified one where the estimated location of the stress axis is typically confined in a limited area. It is clear that the use of the original FPFIT version when a limited number of observations are available would result in a very ambiguous assessment of the principal stress axes orientation for Mygdonia (see fig. 4a) and the corresponding type of faulting (fig. 5a). Moreover, the addition of random noise in the P/S amplitude ratio data has no significant effect when the modified version of the FPFIT program is used.

Finally, removal of available data for 1 to 4 stations from the complete data set for the original first-motion approach gives significantly different results, compared to those corresponding to the complete data set. On the other hand, the use of the proposed method always gives a solution without significant changes, compared to the solution provided for the complete data-set, which demonstrates the robustness of the modified approach.

It should be noted that the finally determined fault plane solutions (Table 2) are in excellent agreement with the stress field and the fault planes observed in the Mygdonia basin. Event 1 corresponds to NE-SW trending normal faults in the western Mygdonia -northern Anthemountas basin area, which are observed in this region in addition to the more typical E-W trending normal faults (e.g. Tranos et al., 2004). Event 2 corresponds to the typical E-W trending normal faults found mainly in central Mygdonia, in agreement with previous studies concerning fault plane solutions from both large (e.g. $1978 \mathrm{M}=6.5$ mainshock) and small earthquakes (e.g. Papazachos et al., 1979; Vamvakaris et al., 2003).

\section{ACKNOWLEDGEMENTS}

This work was partially supported by the European Commission Project No. EVG1-CT-200100040 EUROSEIS-RISK.

\section{REFERENCES}

Aki, K. and P. Richards, 1980. Quantitative Seismology. Theory and Methods, Vol. 1, W. H. Freeman and Co., San Francisco, California.

Hardebeck, J. and P. Shearer, 2003. Using S/P amplitude ratios to constrain the focal mechanisms of small earthquakes. Bull. Seism. Soc. Am. 93, 2434-2444.

Kisslinger, $C ., 1980$. Evaluation of $S$ to $P$ amplitude ratios for determining focal mechanisms from regional network observations, Bull. Seism. Soc. Am. 70, 999-1014.

Kisslinger, C., Bowman, J. and K. Koch, 1981. Procedures for computing focal mechanisms from local (SV/P) data, , Bull. Seism. Soc. Am. 71, 1719-1729.

Papazachos, B., Mountrakis, D., Psilovikos, A. and Leventakis, G., 1979. Surface fault traces and fault plane solutions of May-June 1978 major shocks in the Thessaloniki area, Greece. Tectonophysics 53, 171-183.

Reasenberg, P. and D. Oppenheimer, 1985. FPFIT, FPPLOT, and FPPAGE: FORTRAN computer programs for calculating and displaying earthquake fault-plane solutions. U.S Geol. Surv. Open-file rept. 85-739, 109pp.

Tranos, M. D., Meladiotis, I. D. and Tsolakopoulos, E. P., 2004. Geometrical characteristics, scaling properties and seismic behaviour of the faulting of the Chortiatis region and Anthemountas basin (Northern Greece). $5^{\text {th }}$ International Symposium on Eastern Mediterranean Geology, Thessaloniki (extended abstract).

Vamvakaris, D.A., Papazachos, C.B., Savvaidis, P.D., Tziavos, I.N., Karagianni, E.E., Scordilis, E.M. and Hatzidimitriou, P.M. Stress-field and time-variation of active crustal deformation in the Mygdonia basin based on the joined interpretation of seismological, neotectonic and geodetic data, EGS-AGU-EUG Joint Assembly, Nice, France, April 2003. 